\title{
Cost-Optimal Model Predictive Scheduling of Freezers
}

\author{
Roland Bálint ${ }^{\mathrm{a}, *}$, Attila Fodor ${ }^{\mathrm{a}}$, Katalin M. Hangos ${ }^{\mathrm{a}, \mathrm{b}}$, Attila Magyar ${ }^{\mathrm{a}}$ \\ ${ }^{a}$ Department of Electrical Engineering and Information Systems, Faculty of Information \\ Technology, University of Pannonia, Egyetem str. 10., Veszprém, H-8200, Hungary \\ ${ }^{b}$ Process Control Research Group, HAS Research Institute for Computer Science and \\ Control, Kende str. 13-17., Budapest, H-1111, Hungary
}

\begin{abstract}
A cost-optimal model predictive scheduling algorithm is presented that operates in a day-ahead market. The underlying optimizer is a heuristic branch and bound algorithm that finds the constrained optimal scheduling of a freezer with respect to hourly changing energy price. The method is also able to iteratively re-estimate the heat capacity of the freezer.

Simulation experiments were performed on a freezer model identified from measurement data. Results show that the proposed algorithm successfully decreased the cost of operation, however the computational complexity increases when the price is growing.

The proposed method can be generalized for home appliances of different kind.

Keywords: Smart grids; Demand side management; Model predictive control; Heuristics; Scheduling algorithms
\end{abstract}

\section{Introduction}

One of the major technical challenges nowadays is the efficient management of energy production and consumption. Facing the constrained energy resources and energy production capacity together with the rapidly increasing

\footnotetext{
* Corresponding author

Email addresses: balint.roland@virt.uni-pannon.hu, fax: +36 88624526 (Roland Bálint), foa@almos.uni-pannon.hu (Attila Fodor), hangos.katalin@virt.uni-pannon.hu (Katalin M. Hangos), magyar.attila@virt.uni-pannon.hu (Attila Magyar)
} 
5 and dynamically changing energy consumption, electrical energy providers and line operators, and also the electrical appliances themselves are providing more and more smart solutions with economical, technical and environmental goals, that facilitates the development of smart grid technologies and solutions both on the demand and on the supplier sides.

The approaches from the supplier side include the methods of optimized pricing Joe-Wong et al. (2012) that aim at balancing the electrical grid subject to variations in the supply (e.g. caused by the changing availability of renewable energy sources), and also in the demand. As a result of optimized pricing, hourly changing electrical energy prices are available for the day-ahead electricity market (see e.g. Spot $(2010)$ ), that is continuously expanding, and the amount of energy being traded through them is increasing. The authors of Tianhu et al. (2017) analyzed the effect and potential contribution capability of microgrid to electric power system demand-supply adjustment in hour-ahead electricity market through Price-Based demand response and they concluded Kim et al. (2015) a new framework has been proposed considering decentralized energy coordination and generation that can be utilized in energy dispatch or energy flow scheduling.

The demand side tools and techniques of energy management are also developing rapidly. This area includes the optimal operation of certain electrical appliances with controllable on/off switching taking into account the dynamically changing electrical energy prices and the operating constraints. An optimal day-ahead microgrid scheduling method for an office building considering weather scenarios is developed in Shimomachi et al. (2014), while an optimal residential load control method with price prediction is reported in the paper Mohsenian-Rad and Leon-Garcia (2010). Household appliances can also be a subject of optimal operation or scheduling, see e.g. the paper $\mathrm{Du}$ and Lu (2011). A method is proposed in Báez-González et al. (2016) to minimize the energy cost associated to olive oil production in a day-ahead market.

The optimal energy demand management tasks with changing electrical en- 
ergy prices most often lead to model based optimization problems for which efficient solution methods are available in the literature. In Sou et al. (2011) a mixed integer linear programming based approach is used for the optimal scheduling of domestic appliances in a smart environment. The use of coop40 erative particle swarm optimization and similar soft computing methods was reported in Pedrasa et al. (2010) to energy consumption optimization in smart home applications. An optimal scheduling algorithm has been given in Setlhaolo and Xia (2014) where the optimal scheduling of different household appliances has been formulated as a nonlinear integer programming problem and solved by genetic algorithm. An adaptive scheme has been proposed for temperature control in household freezers with low-end sensing and actuation equipment in Leva et al. (2010).

On the other hand, model predictive control (MPC) is a powerful and popular method for solving multivariable optimal control problems in energy related et al. (2017)). The online estimation of the model parameters (e.g. in a varying temperature situation) enables the controller to adjust the used model to the actual system, e.g. in the work Pedersen et al. (2017), where the demand response potential of a refrigerator system being used in a supermarket has 55 been proposed together with an estimator of the actual food temperature. The authors of Elliott and Rasmussen (2013) propose a decentralized MPC architecture for a multi evaporator-air conditioning system that is decentralized and modular, in order to avoid competing controllers and the practical difficulty of implementing a centralized controller. Model predictive control can also be used in the charging control of electrical vehicles in a Smart Grid as presented in Di Giorgio et al. (2014). The model predictive approach, however, requires to have a reliable dynamical model of the controlled dynamical system. The authors of the work Sossan et al. (2016) propose a grey-box modeling approach for household refrigerators as a basis of a demand side management application.

65 In Schné et al. (2014), the authors provide a dynamical model of a household refrigerator together with a parameter identification. 
The general aim of this work is to propose a theoretically sound yet computationally effective method for cost optimal adaptive scheduling of cooling/heating appliances. Because of the computationally exhaustive nature of the standard MPT toolbox Herceg et al. (2013) available for this purpose (see in Bálint and Magyar (2016)), heuristic elements were needed to develop a computationally feasible method Bálint et al. (2017b). In addition, the need for an adaptive version of the method has also arisen to follow the change in the load of the cooling/heating appliances Bálint et al. (2017a). Based on the earlier attempts

75 Bálint et al. (2017a), this paper proposes an effective method for cost optimal adaptive scheduling of cooling appliances together with a simple way of estimating the model parameters needed for the scheduling.

The paper is organized as follows. The problem is defined in Section 2 together with the dynamical model of the freezer. The scheduling problem is

80 formulated as a model predictive control problem in Section 3 , two proposed heuristic algorithms are also presented here. The case study of a simple freezer is given in Section 4, which is followed by the discussion of the results in Section 5. Finally, the most important concluding remarks and some future research directions are given in Section 6 .

85 2. Problem statement

The problem of cost-optimal scheduling of freezers possesses important specialties that can be effectively utilized in the proposed heuristic solution. These specialties are present both in the dynamic models of the freezers and in the special control aim driven by the time-dependent electricity prices.

90 2.1. Dynamic model of freezers for scheduling

In the simplest case, a freezer can be regarded as a container that is cooled by a cooling liquid circuit driven by an electrical motor. The schematic picture of the main elements of this simple freezer is shown in Fig. 1 .

The containment is characterized by its air temperature $T_{a}$. It is heated 95 by the outer environment through the door of the freezer, and cooled by the 


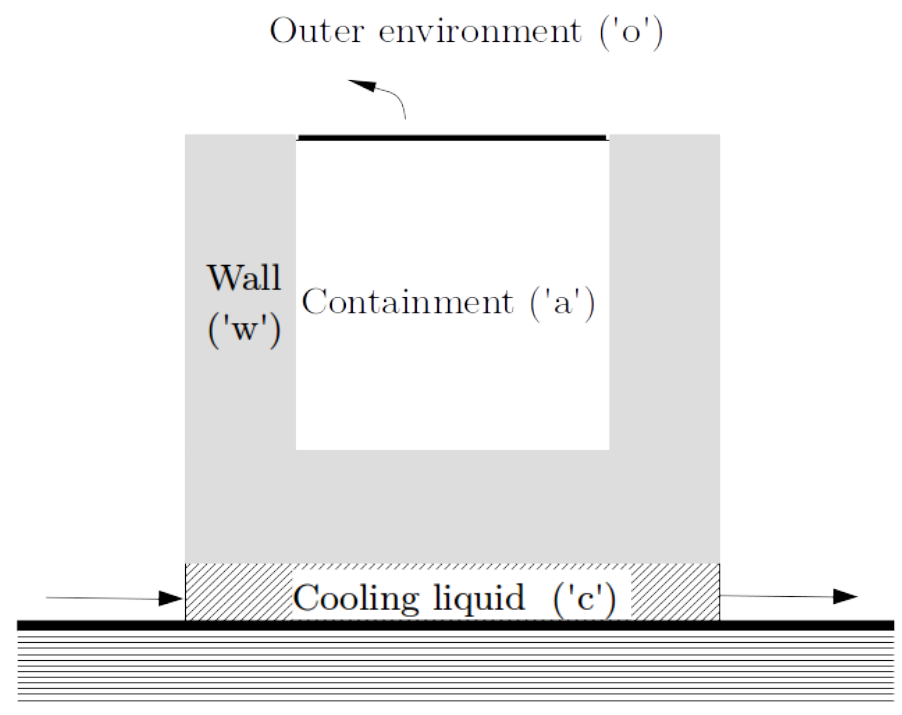

Figure 1: The schematic picture of the freezer

wall with temperature $T_{w}$. A liquid cooling system with liquid temperature $T_{c}$ provides cooling when the cooling binary switch $S$ is on, while there is no cooling of the wall when it is switched off $(S=0)$. The side wall is also heated by the outer environment.

The variables and parameters of the freezer model are collected in Table 1

The engineering model. The simplest possible dynamic model that describes the dynamics of the above described freezer can be constructed from the energy balances for the containment air and that of the wall in the following form (see Hangos and Cameron (2001))

$$
\begin{array}{r}
C_{a} \frac{d T_{a}}{d t}=K_{w}\left(T_{w}-T_{a}\right)+K_{o}\left(T_{o}-T_{a}\right) \\
C_{w} \frac{d T_{w}}{d t}=K_{w}\left(T_{a}-T_{w}\right)+K_{x}\left(T_{o}-T_{w}\right)+S \cdot K_{c}\left(T_{c}-T_{w}\right)
\end{array}
$$

with the variables and parameters collected in Table 1.

The first terms in the right-hand side of the equations correspond to the heat 
Table 1: Model variables and parameters

\begin{tabular}{lccc} 
meaning & symbol & classification & unit \\
\hline containment air temperature & $T_{a}$ & state variable & ${ }^{\circ} \mathrm{C}$ \\
wall temperature & $T_{w}$ & state variable & ${ }^{\circ} \mathrm{C}$ \\
binary switch status & $S$ & input variable & - \\
outer air temperature & $T_{o}$ & parameter & ${ }^{\circ} \mathrm{C}$ \\
cooling liquid temperature & $T_{c}$ & parameter & ${ }^{\circ} \mathrm{C}$ \\
minimal inner air temperature & $T_{a, \text { min }}$ & parameter & ${ }^{\circ} \mathrm{C}$ \\
maximal inner air temperature & $T_{a, \text { max }}$ & parameter & ${ }^{\circ} \mathrm{C}$ \\
minimal wall temperature & $T_{w, m i n}$ & parameter & ${ }^{\circ} \mathrm{C}$ \\
maximal wall temperature & $T_{w, m a x}$ & parameter & ${ }^{\circ} \mathrm{C}$ \\
air-wall heat transfer coeff. & $K_{w}$ & parameter & $\frac{\mathrm{kW}}{{ }^{\circ} \mathrm{C}}$ \\
air-env. heat transfer coeff. & $K_{o}$ & parameter & $\frac{\mathrm{kW}}{\circ} \mathrm{C}$ \\
wall-env. heat transfer coeff. & $K_{x}$ & parameter & $\frac{\mathrm{kW}}{{ }^{\circ} \mathrm{C}}$ \\
wall-cool. liq. heat transfer coeff. & $K_{c}$ & parameter & $\frac{\mathrm{kW}}{\circ} \mathrm{C}$ \\
heat capacity of the containment & $C_{a}$ & parameter & $\frac{\mathrm{kJ}}{\circ}$ \\
heat capacity of wall & $C_{w}$ & parameter & $\frac{\mathrm{kJ}}{{ }^{\circ} \mathrm{C}}$ \\
\hline
\end{tabular}

transfer between the containment air and the wall, the second transfer terms correspond to the transfer between the outer environment and the containment air or the wall, respectively, and the last term in the second equation describes the effect of the cooling liquid. The parameters of the model are assumed to be constant.

The state and input variables. Now we can identify the state $(\boldsymbol{x})$ and input variables $(\boldsymbol{u})$ of the dynamic model as follows:

$$
\boldsymbol{x}=\left[\begin{array}{c}
T_{a} \\
T_{w}
\end{array}\right], \quad \boldsymbol{u}=S
$$

where $S$ is the state of the switch.

Piecewise affine model. Let us define two operating modes of the freezer: the cooling and the reheating modes. In both cases the state space model is in the 
standard linear time-invariant affine model form

$$
\begin{aligned}
& \dot{\boldsymbol{x}}=\boldsymbol{A} \boldsymbol{x}+\boldsymbol{B} \boldsymbol{u}+\boldsymbol{f} \\
& \boldsymbol{y}=\boldsymbol{C} \boldsymbol{x}
\end{aligned}
$$

but the value of the coefficient matrices $\boldsymbol{A}, \boldsymbol{B}, \boldsymbol{C}$ differ.

Cooling dynamics. The first case is when the switch is closed $(S=1)$, i.e. the refrigerator is cooling. Then the parameter matrices and vectors are

$$
\begin{aligned}
& \boldsymbol{A}_{\text {on }}=\left[\begin{array}{cc}
-\frac{K_{w}+K_{o}}{C_{a}} & \frac{K_{w}}{C_{a}} \\
\frac{K_{w}}{C_{w}} & -\left(\frac{K_{w}}{C_{w}}+\frac{K_{c}}{C_{w}}+\frac{K_{x}}{C_{w}}\right)
\end{array}\right], \\
& \boldsymbol{B}_{\text {on }}=\left[\begin{array}{c}
0 \\
\frac{T_{c} K_{c}}{C_{w}}
\end{array}\right], \boldsymbol{f}_{\text {on }}=\left[\begin{array}{c}
\frac{K_{o} T_{o}}{C_{a}} \\
\frac{K_{x} T_{o}}{C_{w}}
\end{array}\right], \boldsymbol{C}=\left[\begin{array}{ll}
1 & 0 \\
0 & 1
\end{array}\right] .
\end{aligned}
$$

Reheating dynamics. The second case is when the switch is open $(S=0)$, i.e. the freezer is reheated to the environmental temperature. Then the parameter matrices and vectors are as follows:

$$
\begin{aligned}
& \boldsymbol{A}_{\text {off } f}=\left[\begin{array}{cc}
-\frac{K_{w}+K_{o}}{C_{a}} & \frac{K_{w}}{C_{a}} \\
\frac{K_{w}}{C_{w}} & -\left(\frac{K_{w}}{C_{w}}+\frac{K_{x}}{C_{w}}\right)
\end{array}\right], \\
& \boldsymbol{B}_{\text {off }}=\left[\begin{array}{l}
0 \\
0
\end{array}\right], f_{\text {off }}=\left[\begin{array}{l}
\frac{K_{o} T_{o}}{C_{a}} \\
\frac{K_{x} T_{o}}{C_{w}}
\end{array}\right], C=\left[\begin{array}{ll}
1 & 0 \\
0 & 1
\end{array}\right] .
\end{aligned}
$$

More details on the engineering model together with a parameter set relevant to a household refrigerator can be found in Bálint et al. (2017b).

\subsection{Changing energy prices on a day-ahead market}

In the modern power grid the day-ahead market serves as the marketplace for trading power. The service provider gives the electricity price, i.e. the price for electrical energy, for the next 24 hours. Fig. 2 shows the hourly electricity 
prices for a week, where each line corresponds to the prices of a day. There is supposed to be no uncertainty in the energy price in the day-ahead period, i.e. the price is always supposed to be known for the next 24 hours. As it



Figure 2: Electricity price of a day-ahead market for a week. Source: Spot (2010)

is apparent in Fig. 2, the energy price of the same period for different days is highly fluctuating, so the price-optimal operation of home appliances in a day-ahead market can be formulated as an optimal scheduling problem (see in Joe-Wong et al. (2012)). The real data used for the simulations are obtained from the report of Spot (2010).

Operating cost. Given the dynamically changing but known-ahead price for electrical energy consumed by the cooling electrical motor (denoted by $p(t)$ ) as a function of time $t$, the aim of the control is to minimize the operating cost, that is in the following general form

$$
\int_{\tau=0}^{24 h}(p(\tau) \cdot S(\tau)) d \tau
$$


The above integral computes the operating cost using the on/off status of the switch, the electrical power of the motor and the time-dependent cost of electrical energy in continuous time.

\section{Optimal scheduling of freezers}

The control aim, i.e. to minimize the operating cost drawn up in subsection 2.2 suggests the use of model predictive control theory for this problem. Moreover, since the control law is the switching sequence of the freezers binary input, the problem to be solved is a constrained optimal scheduling problem.

\subsection{Model predictive control and scheduling}

In order to be able to apply the tools of model predictive control theory, one needs a suitable predictive model that describes the behavior of the controlled plant (freezer). The cost function usually formed as a combination of quality and/or economic expectations against the system, moreover, constraints are also introduced to the system's (state) variables which usually define some regions of safety operation for the system.

System model. As a first step the continuous-time model $6 \sqrt[6]{9}$ is discretized with sampling time $h$ in order to get the discrete-time piecewise affine (PWA) system model used in the sequel.

$$
\Sigma_{i}:\left\{\begin{array}{rl}
\boldsymbol{x}_{k+1} & =\boldsymbol{\Phi}_{\boldsymbol{i}} \boldsymbol{x}_{k}+\boldsymbol{\Gamma}_{\boldsymbol{i}} u_{k}+h \boldsymbol{f}_{i} \\
\boldsymbol{y}_{k} & =\boldsymbol{C} \boldsymbol{x}_{k}
\end{array}, i \in\{\text { on } ; \text { of } f\},\right.
$$

where $\boldsymbol{x}_{k}$ stands for the value of the vector valued signal $\boldsymbol{x}$ at the discrete time instant $k$, matrices $\boldsymbol{\Phi}=\boldsymbol{e}^{\boldsymbol{A} \boldsymbol{h}}$ and $\boldsymbol{\Gamma}=\boldsymbol{A}^{-1}\left(\boldsymbol{e}^{\boldsymbol{A} \boldsymbol{h}}-\boldsymbol{I}\right) \boldsymbol{B}$ are the state- and input matrices of the state equation discretized by sampling time $h$, and $\boldsymbol{f}$ is the constant vector in the continuous time model.

Cost function. The operating cost to be minimized (i.e. the price of the consumed electrical energy) is the discrete sum 12 which can be regarded as 
an approximation of 10 . Although the sampling time $h$ is kept considerably smaller than one hour (i.e. the sampling time of the price) the values of 10 and $(12)$ may be different.

$$
\operatorname{cost}=\sum_{j=1}^{N} p_{j} u_{j} h
$$

where $N$ is supposed to be the prediction horizon size. It is supposed that the price levels of the next day are known at least $H=N h$ time (prediction time) before midnight.

Constraints. The state constraints of the form 13 can be used directly in the MPC framework.

$$
\underline{\boldsymbol{x}} \leq \boldsymbol{x}_{k} \leq \overline{\boldsymbol{x}}
$$

where the lower and upper bounds of the state variables are evaluated componentwise. The freezer air and wall temperatures has to obey the following constraints (14).

$$
\underline{\boldsymbol{x}}=\left[\begin{array}{c}
T_{a, \min } \\
T_{w, \min }
\end{array}\right], \quad \overline{\boldsymbol{x}}=\left[\begin{array}{c}
T_{a, \max } \\
T_{w, \max }
\end{array}\right] .
$$

Summarized, the MPC problem is to minimize 12 in $u_{k}$ with respect to (11) and the constraints (13). In each iteration the optimization of the cost 12 . is performed from the actual time to a fixed size prediction horizon $H=N h$, and the first element of the optimizing input sequence is applied to the real system.

\subsection{Heuristic optimal scheduling algorithm}

The integer (binary) variables in the optimization problem to be solved during the model predictive scheduling formulated in Section 3.1 makes it difficult to solve the problem using off-the-shelf tools Herceg et al. (2013). An effective heuristic scheduling algorithm is proposed in this section that can be used as the optimizer of the MPC problem. 
Heuristics. The proposed algorithm is a version of branch and bound type optimization where the branch step introduces possible switching sequences and the bound step decreases the size of the solution space based on the following three heuristic rules Bálint et al. (2017b):

Rule 1: Any scheduling sequence that yields an $\boldsymbol{x}$ breaking the bounds $(13)$ is not allowed.

Rule 2: Between any two scheduling sequences the one yields a higher $\boldsymbol{x}$ at a higher cost is not optimal.

Rule 3: Any scheduling sequence containing a cooling step that could have been performed later for a lower energy price is not optimal.

The first two rules are easy to implement. The idea to apply Rule 3 is the preliminary determination of price-equivalent cooling time $t_{i}^{p}$ for all subsequent price periods of the day. For the $i^{t h}$ time period (hour), it can be calculated as

$$
t_{i}^{p}=\left\lceil\frac{p_{i+1}}{p_{i}} h\right\rceil, \quad i=1, \ldots, 24,
$$

where $p_{i}$ is the price for the $i^{\text {th }}$ time interval and $\lceil$.$\rceil represents the ceiling$ function.

Using the price-equivalent cooling times 15 , it is possible to determine the reference states used in Rule 3 as follows. Off-line dynamical simulations of (11) 175 are performed from the initial state $\overline{\boldsymbol{x}}$ for a times $t_{i}^{p}$, respectively. The final state of the simulations are denoted by $\boldsymbol{x}_{i}^{r}$ and can be used as the reference values of the comparison: If the actual state $\boldsymbol{x}<\boldsymbol{x}_{i}^{r}$ during the period $t_{i}^{p}$ then switching the cooling on yields a suboptimal sequence.

The pseudo code of the proposed optimal heuristic scheduling procedure HeuristicScheduler() is given in Algorithm 1. The two procedures used in Algorithm 1 are described as follows. Procedure DeleteRow $(K, i)$ simply deletes the $i^{\text {th }}$ row of matrix (or vector) $K$. Procedure FindMinimalElement $(s)$ returns the minimal element of the vector $s$. 


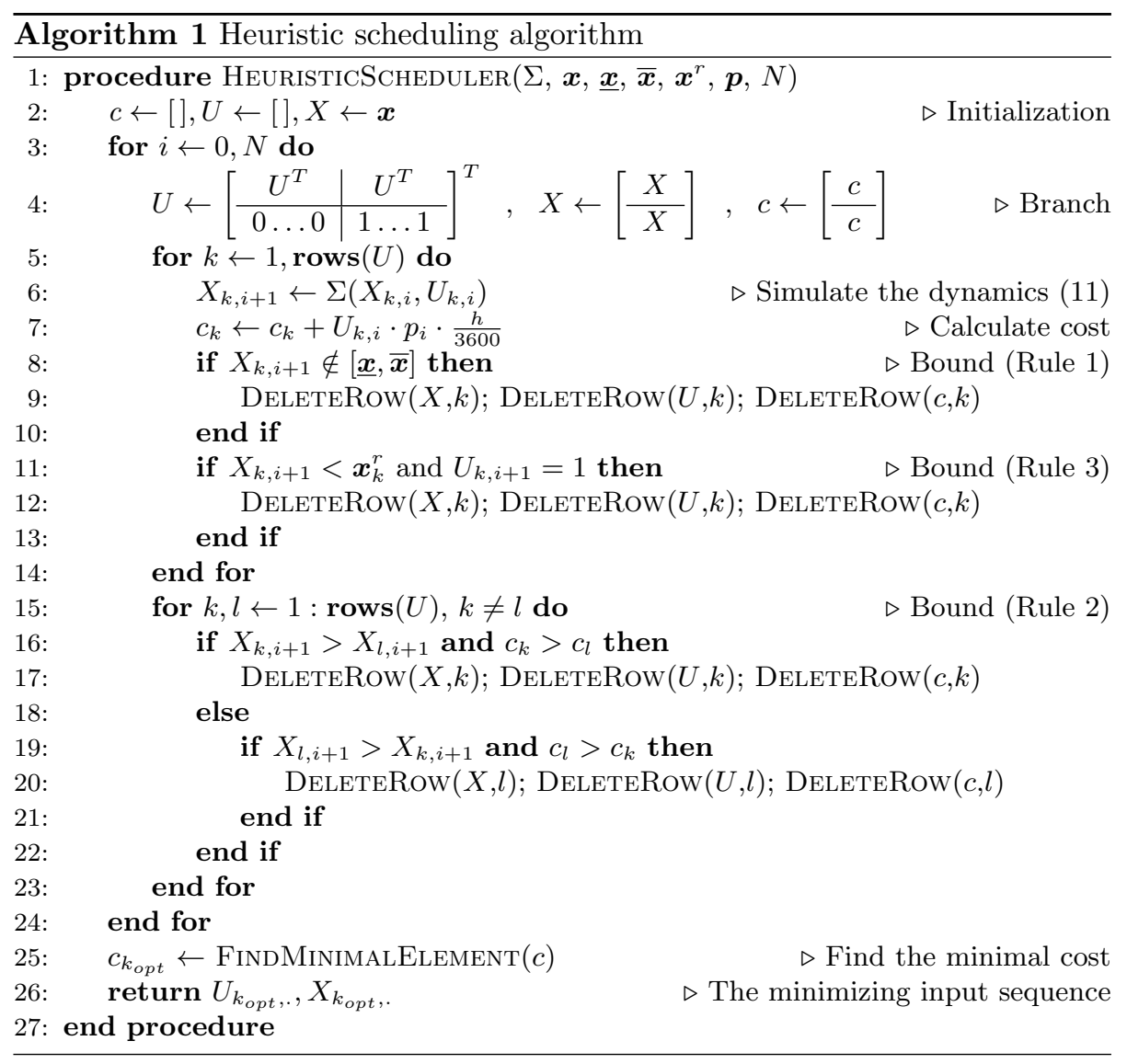


It is important to note, that the calculation of $t_{i}^{p}$ and $\boldsymbol{x}_{i}^{r}$ can be calculated

$$
\text { scheduling sequence. }
$$

The key parameter behind the adaptivity is the heat capacity $C_{a}$ of the interior air since this compartment contains the goods. The actual heat capacity $C_{a}^{a c t}$ is supposed to vary between a minimal value $C_{a_{m i n}}$ that corresponds to the empty freezer and a maximal value $\left(C_{a_{\max }}\right)$. The change in the heat capacity can only be detected from the available temperature measurements $\left(T_{a}\right)$ taking place in the freezer. The adaptivity of the model predictive scheduler is implemented as a parameter estimation step in which an estimate $C_{a}^{e s t}$ the actual value of the freezer interior heat capacity is being determined based on the available temperature measurements. The sensitivity of the proposed adaptive method depends on a predefined temperature difference limit $(\Delta T)$. The heat capacity is re-estimated every iteration when

$$
\left|T_{a}(n)-T_{a}^{m}(n)\right|>\Delta T
$$

where $T_{a}(n)$ is the air temperature of the model 11 and $T_{a}^{m}(n)$ denotes the measured value of the air temperature at the $n$-th time instant, respectively.

The pseudo code of the proposed adaptive scheduler is given in Algorithm 2 , where input parameter $\Sigma$ denotes the PWA dynamics (11). The main difference between the previously presented Algorithm 1 and Algorithm 2 is the fact, that Algorithm 2 performs an online parameter estimation step in the beginning of the control loop and calls Algorithm 1 as a subroutine. The online estimation of 


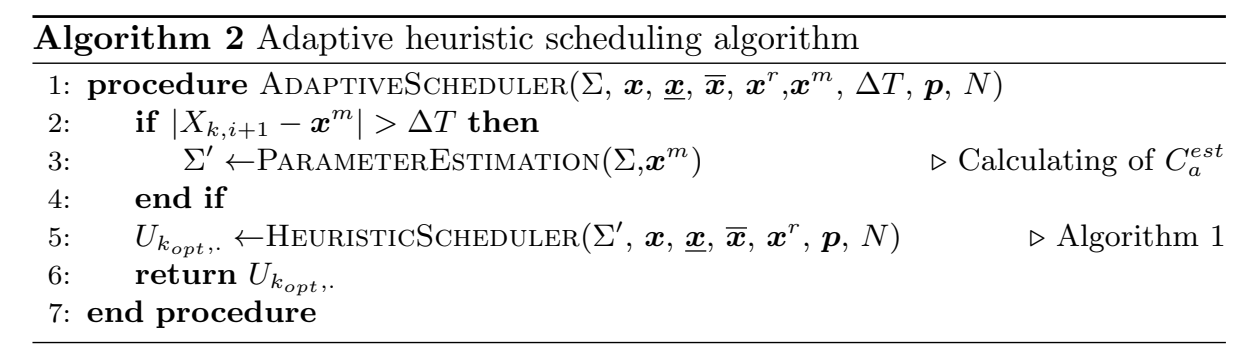

Online estimation of $C_{a}$. The online estimation of $C_{a}$ (denoted by PARAMETEREstimation() in Algorithm 2) is performed at the beginning of a control cycle if the difference between the model output temperature and the measured temperature $\boldsymbol{x}^{m}$ is greater than the predefined $\Delta T$ value (see condition $(16)$ ). The estimation is basically a bisection method used for finding the root of the difference $\Delta T_{a}\left(C_{a}\right)$ with respect to the heat capacity parameter $C_{a}$ (see Bálint et al. (2017a) for details).

$$
\Delta T_{a}\left(C_{a}\right)=T_{a}^{m}(n)-T_{a}\left(C_{a}, n\right)
$$

where $T_{a}\left(C_{a}, n\right)$ is the containment air temperature computed from the discretized version of model 11 . The root of 17 is denoted by $C_{a}^{e s t}(n)$.

The search interval for the value of $C_{a}^{e s t}(n)$ is an interval of length $10 C_{a_{\text {min }}}$ with one of the end points being the previous guess for the interior heat capacity, i.e. $C_{a}^{e s t}(n-1)$. The other end point of the search interval is $C_{a}^{e s t}(n-1) \pm$ $10 C_{a_{m i n}}$ depending on the sign of the difference $T_{a}(n)-T_{a}^{m}(n)$. The number of necessary iterations of the above bisection algorithm depends on the desired tolerance $\varepsilon$ in the following form

$$
N_{C_{a}}=\log _{2}\left(\frac{10 C_{a_{m i n}}}{\varepsilon C_{a_{\min }}}\right)=\log _{2}\left(\frac{10}{\varepsilon}\right) .
$$




\subsection{The steps of the adaptive model predictive scheduling method}

As a summary of the above, the following steps should be carried out when

205 using the proposed scheduling method on a new freezer system.

1. Calibrating the dynamic model before real-time application using the old hysteresis type on-off control, with nominal load (see section 4.1 of the Case study).

2. Sensitivity analysis with respect to parameter uncertainties should be carried out using the method described in Bálint et al. (2017b).

3. Determination of the minimal necessary prediction horizon size by using simulation (see section 4.2 in the Case study)

4. Real-time execution of the adaptive scheduling algorithm that adapts the operation to the changing load conditions. (see section 4.3 in the Case study)

\section{Case Study}

A simple case study is used to illustrate the use and the properties of the the proposed cost-optimal scheduling method. A simple freezer (RIO S-68) for storing ice-cream in a shop was used, the parameters of which were estimated 220 using measured data. This model was used to illustrate the operation and properties of the scheduling algorithms.

\subsection{Freezer modeling and identification}

The first step of the model predictive scheduling is to develop a reliable model of the system, in the present case a freezer, to be scheduled.

Freezer description

A relatively small freezer operating in a grocery shop is chosen for the case study, that is used for storing ice cream. The containment volume of the freezer is approximately $0.3 \mathrm{~m}^{3}$ with $9.5 \mathrm{~kg}$ ice cream stored in it during the experiments. 
The temperature of the containment $T_{a}$ and that of the wall $T_{w}$ was measured in an automatic temperature regulating mode keeping the $-27^{\circ} \mathrm{C}$ setpoint using a conventional hysteresis type on/off controller. The actual status (on/off) of the switch $S$ was also recorded. The electric power of the cooling motor was $240 \mathrm{~W}$.

\section{Parameter estimation}

The piecewise affine model described in subsection 2.1 was used for the parameter estimation.

Estimation method. A quadratic loss function characterizing the measure of fit was used in the following form:

$$
V(\theta)=\int_{t=t_{0}}^{t_{f}} w_{a}\left(T_{a}(t)-\hat{T}_{a}(t)\right)^{2}+w_{w}\left(T_{w}(t)-\hat{T}_{w}(t)\right)^{2} \mathrm{~d} t
$$

where $\hat{T}_{a}$ and $\hat{T}_{w}$ are the model-predicted values of the containment and the wall temperatures, respectively, $\theta$ is the vector of model parameters, and $w_{a}=3$, $w_{w}=1$ are weighting factors.

The Matlab function fminsearch was used for minimizing the above loss function with respect to the parameter values. A plausibility region was given to each parameter based on physical insight that was taken into account during the optimization.

Estimation results. Together with the estimated value of the parameters, the value of the loss function (19) was also computed as a function of the possible parameter values. The level set curves of the loss function were also investigated and evaluated in order to gain information about the correlation of the estimated parameter values and about sensitivities with respect to parameter variations.

The results indicated that the estimates of some parameter pairs, for example of $\left(T_{c}, K_{c}\right)$ are highly correlated, in this case physical insight was used to choose 
the actual value of one of them. Using this regularization, the estimated values shown in Table 2 were obtained.

Table 2: Estimated freezer parameter set

\begin{tabular}{|c|c|c|c|}
\hline meaning & symbol & estimated value & unit \\
\hline cooling liquid temperature & $T_{c}$ & -43.6 & ${ }^{\circ} \mathrm{C}$ \\
\hline air-wall heat transfer coeff. & $K_{w}$ & 0.0241 & $\frac{\mathrm{kW}}{{ }^{\circ} \mathrm{C}}$ \\
\hline air-env. heat transfer coeff. & $K_{o}$ & 0.0021 & $\frac{\mathrm{kW}}{{ }^{\circ} \mathrm{C}}$ \\
\hline wall-env. heat transfer coeff. & $K_{x}$ & 0.0186 & $\frac{\mathrm{kW}}{{ }^{\circ} \mathrm{C}}$ \\
\hline wall-cool. liq. heat transfer coeff. & $K_{c}$ & 0.173 & $\frac{\mathrm{kW}}{{ }^{\circ} \mathrm{C}}$ \\
\hline heat capacity of the containment & $C_{a}$ & 40.1 & $\frac{\mathrm{kJ}}{{ }^{\circ} \mathrm{C}}$ \\
\hline heat capacity of wall & $C_{w}$ & 71.4 & $\frac{\mathrm{kJ}}{{ }^{\circ} \mathrm{C}}$ \\
\hline
\end{tabular}

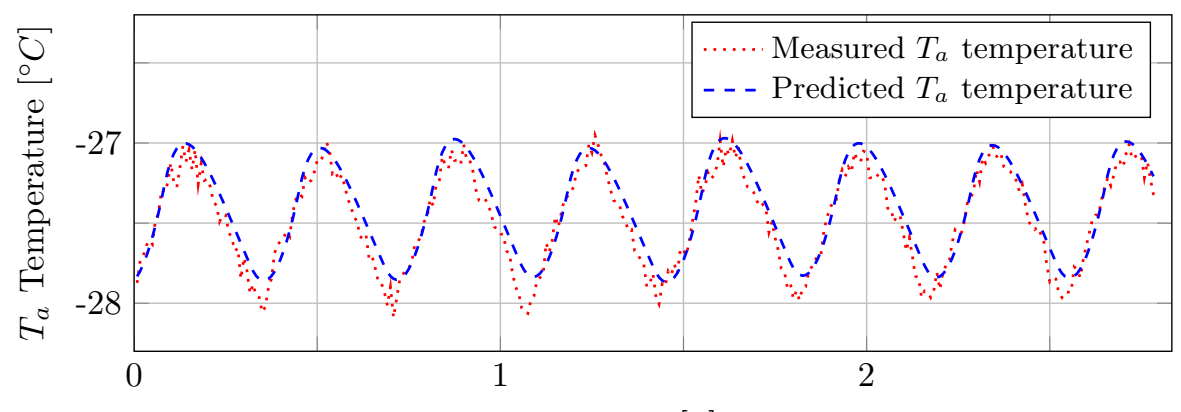

Time $[h]$

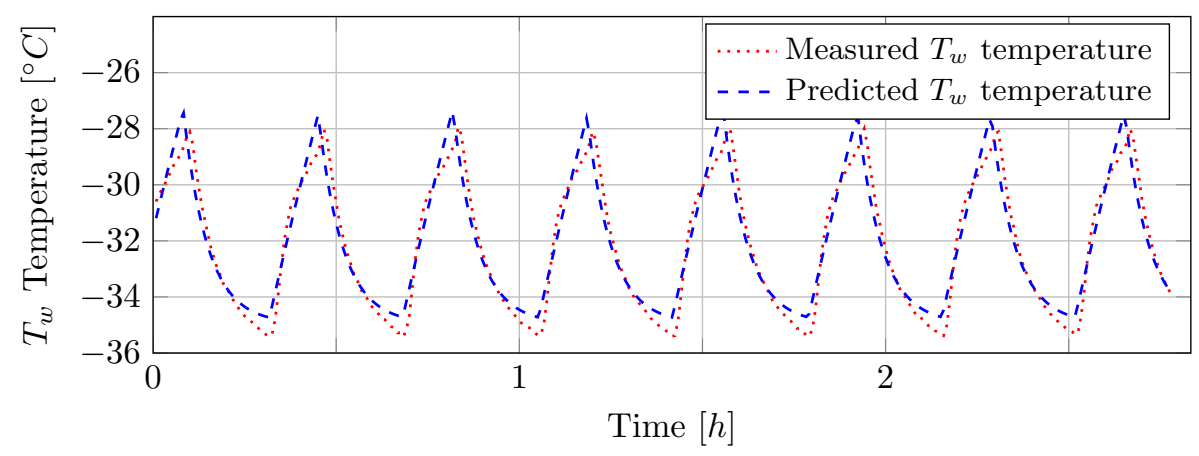

Figure 3: The measured and model predicted temperatures

The quality of the estimation is characterized by plotting the measured containment and wall temperatures against their model predicted values using the estimated parameter values in the model. In Fig. 3 a good agreement of the 
measured and model predicted temperatures are shown that indicates the good quality of the model for model predictive scheduling purposes. Of course, a better prediction quality could be obtained with a more detailed an sophisticated model but the aim of this model is to catch the qualitative dynamical behavior of the system with the simplest possible dynamics. The simplicity of the model is crucial from the implementation point of view (e.g. on a MCU).

\section{Sensitivity investigations}

We investigated the sensitivity of the model predicted temperatures with respect to the critical model parameter, the heat capacity of the containment $C_{a}$. This parameter depends on the mass and specific heat of the actual content, that is the goods stored in the freezer (in this case the ice cream). This parameter may change in time depending on the load and consumption of the stored freezer content.

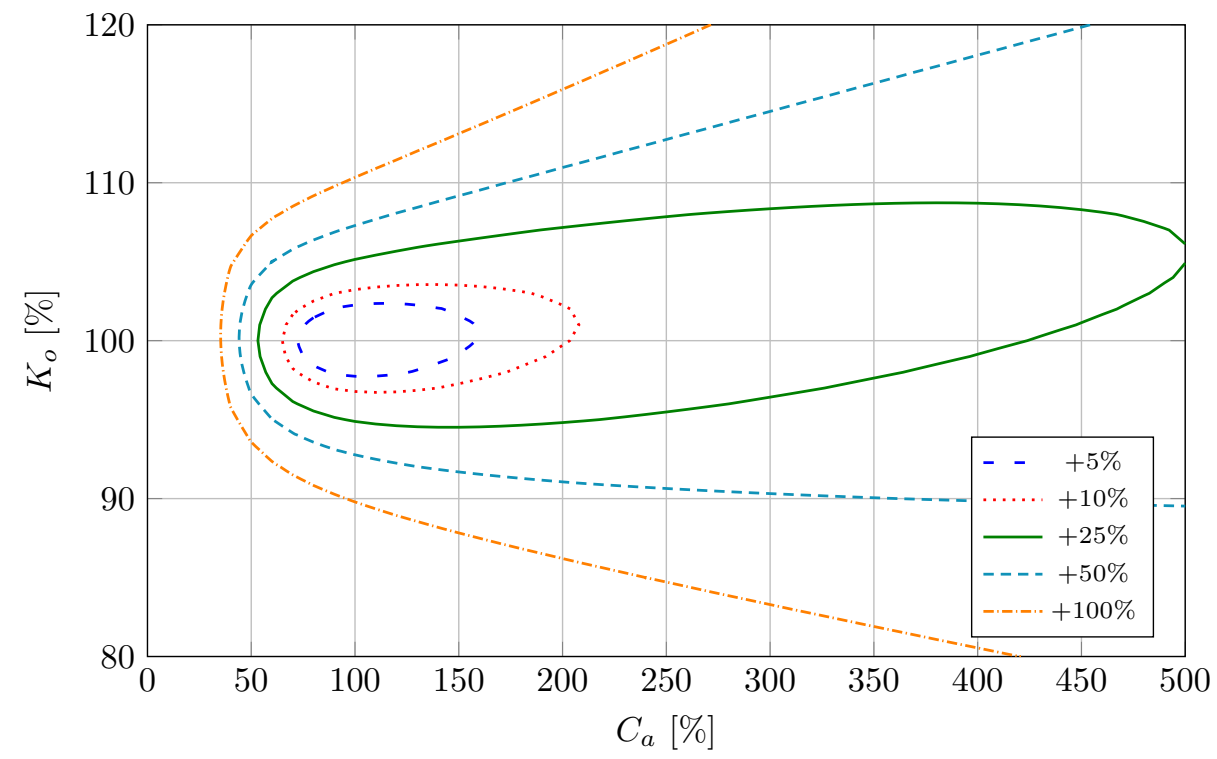

Figure 4: The level set curves of the loss function with parameters $K_{o}$ and $C_{a}$.

The sensitivity analysis shows that the estimated value of $C_{a}$ is independent of the other parameters (no high correlation is observed), and it influences 
critically the model response. Fig. 4 shows the level set curves of the loss function in the parameter sub-space for the parameters $K_{o}$ and $C_{a}$. A definite sharp minimum is observed, that shows the strong influence of $C_{a}$ on the loss.

\subsection{Heuristic model predictive scheduling}

The aim of the first set of simulation experiments were to investigate the effect of prediction horizon size $(H)$ on the performance of Algorithm 1 . In order to make it difficult for the model predictive scheduler to find the optimal solution, Wednesday, i.e. the day with the highest price variability (see Figure 2 has been chosen for the experiments. The comparative simulation results are given in Fig. 5, where the times spent with the solution of the optimization problem at each iteration are depicted for the whole day. The results are in line with the engineering expectations i.e. a larger prediction horizon tends to be computationally more demanding. It can easily be seen in Fig. 5 that $H$ amount of time before the energy price is increasing the optimization problem to be solved gets computationally more demanding.

Table 3 gives a more complete picture of the computational effect of the horizon size. It is apparent that days with smaller price variance (e.g. Monday, see Fig. 22 have a smaller total optimization time (i.e. complexity). It is important to note that from the point of view of the complexity the number of price growth steps is much more important than the degree of price growth. It can be seen that the overall optimization time (i.e. complexity) of a day is in strong correlation with the number of price growth steps.

Another aspect of horizon size has also been investigated, namely the effect of horizon size on the daily price obtained by the model predictive scheduler. Table 4 shows the results of the experiment. The reference values obtained from the classical hysteresis control can be seen in the last row of the table. It is easy to see, that

(i) the size of prediction horizon does not have a serious effect on the daily cost obtained by the algorithm, 

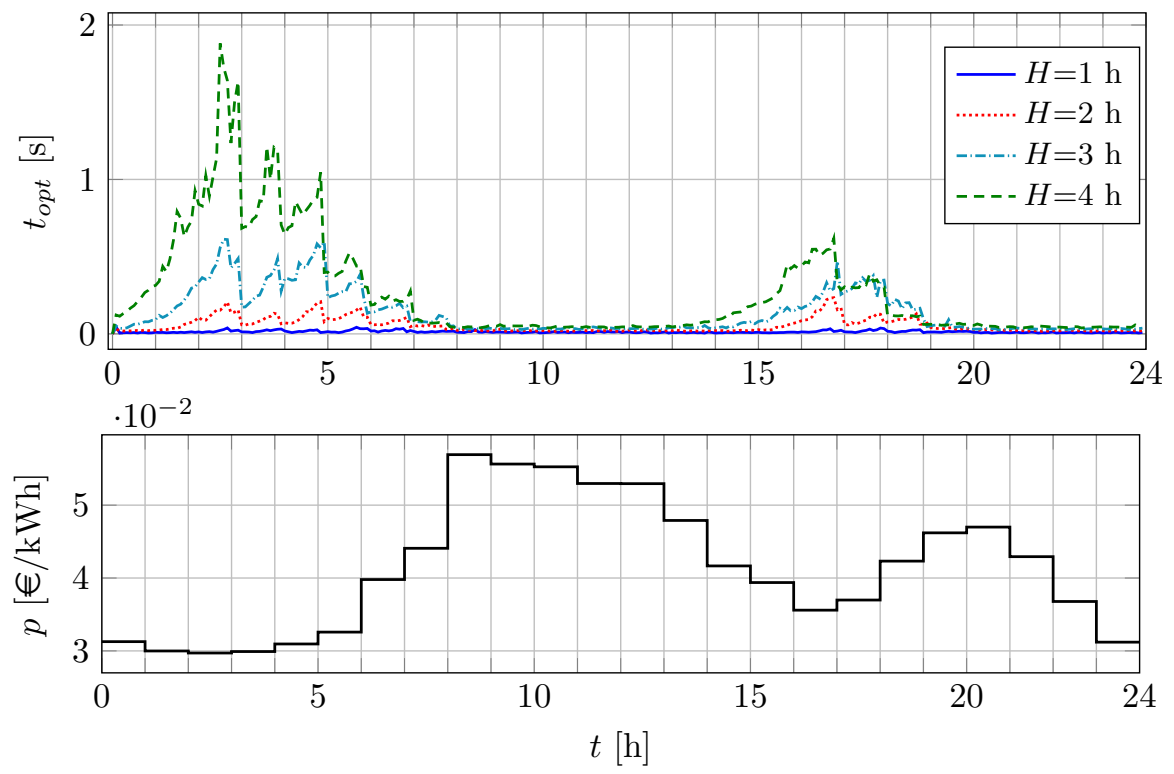

Figure 5: Top: The effect of prediction horizon $H$ on the optimization time $t_{\text {opt }}$. Bottom: Hourly changing energy price for the examined day (Wednesday). It is apparent that $H$ amount of time before the energy price is increasing the optimization problem to be solved gets computationally more demanding.

Table 3: The effect of the prediction horizon size on the cumulative optimization time (given in seconds) for a whole day of the week, i.e. $\sum_{i \in \text { day }} t_{p}(i)$. The number of price growth steps are shown in the last row.

\begin{tabular}{|c|c|c|c|c|c|c|c|}
\hline$H[\mathrm{~h}]$ & Mon. & $\mathrm{Tu}$. & Wed. & Th. & Fri. & Sat. & Sun. \\
\hline 1 & 7 & 10 & 7 & 7 & 8 & 8 & 7 \\
\hline 2 & 30 & 47 & 35 & 32 & 36 & 36 & 26 \\
\hline 4 & 130 & 327 & 168 & 153 & 180 & 187 & 113 \\
\hline 8 & 427 & 2675 & 926 & 731 & 1051 & 914 & 642 \\
\hline 12 & 1023 & 6900 & 1882 & 1381 & 1881 & 2377 & 1213 \\
\hline 24 & 3507 & 26504 & 4743 & 4843 & 5099 & 6637 & 2540 \\
\hline \multicolumn{8}{|c|}{ Number of price increases } \\
\hline & 9 & 17 & 11 & 11 & 14 & 12 & 9 \\
\hline
\end{tabular}

(ii) the proposed method outperforms the classical control method on each of the examined days. 
Table 4: The effect of the prediction horizon size on the daily operating cost $[€ / \mathrm{kWh}]$. As a reference, the daily values yielded by the classical hysteresis control algorithm are shown in the last row.

\begin{tabular}{lccccccc}
$H[\mathrm{~h}]$ & Mon. & Tu. & Wed. & Th. & Fri. & Sat. & Sun. \\
\hline 1 & 0.442 & 0.445 & 0.607 & 0.487 & 0.509 & 0.565 & 0.597 \\
2 & 0.440 & 0.442 & 0.604 & 0.484 & 0.503 & 0.563 & 0.595 \\
4 & 0.442 & 0.445 & 0.607 & 0.487 & 0.506 & 0.566 & 0.595 \\
8 & 0.438 & 0.437 & 0.602 & 0.482 & 0.501 & 0.561 & 0.593 \\
12 & 0.440 & 0.442 & 0.604 & 0.485 & 0.503 & 0.563 & 0.595 \\
24 & 0.440 & 0.445 & 0.604 & 0.485 & 0.503 & 0.563 & 0.595 \\
\hline \multicolumn{7}{c}{ hysteresis (classical) control } \\
\hline$T_{s}=60 s$ & 0.464 & 0.464 & 0.638 & 0.510 & 0.531 & 0.594 & 0.627
\end{tabular}

\subsection{Adaptive scheduling}

the air temperature, $T_{a, \max }$. The plot at the bottom (Fig. 6) shows the actual value of the heat capacity $C_{a}$ that changes due to the changing goods in the freezer, the non-adaptive MPC is supposed to know the heat capacity at the initial time (dashed) while the adaptive algorithm follows the actual value be 


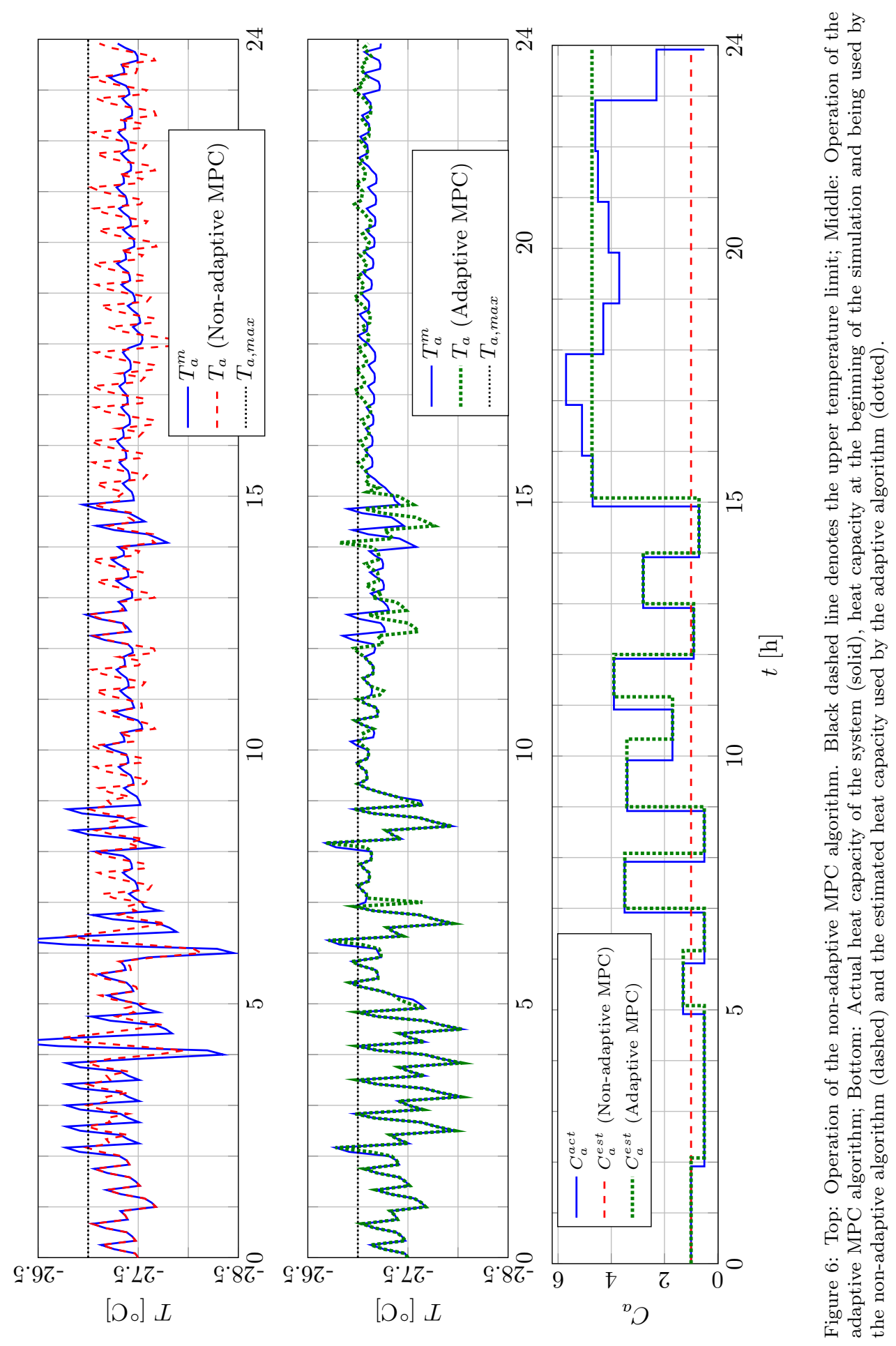


Table 5: The daily operating costs $[€ / \mathrm{kWh}]$ for $H=2$ hours in the case of the nonadaptive and the adaptive algorithms.

\begin{tabular}{lccccccc} 
Algorithm & Mon. & Tu. & Wed. & Th. & Fri. & Sat. & Sun. \\
\hline Nonadaptive & 0.440 & 0.442 & 0.604 & 0.484 & 0.503 & 0.563 & 0.595 \\
Adaptive & 0.430 & 0.430 & 0.589 & 0.474 & 0.491 & 0.550 & 0.580 \\
\hline
\end{tabular}

\section{Discussion}

5.1. Specialties of the problem and their use in choosing the parameters of the algorithm

There are two important special properties of the dynamic model of the freezer (see in Eqs. (1)-(2) in the continuous time, and consequently in Eq. (11) in the discrete time case) that are utilized in developing the heuristic rules in the proposed method (both in the constant and the adaptive cases) 
(i) the model input $S$ is discrete valued, $u(k)=S(k) \in\{0,1\}$,

(ii) the response of the model output variables $T_{a}$ and $T_{w}$ is strictly monotonous

Fig. 3 shows that it is indeed the case. Furthermore, the dominant time constant of the system can also be estimated roughly from the figure, that shows the dynamic response under normal, non-optimized operating conditions. tion of another freezer, the data can be used for choosing the sampling rate and the error tolerance limits of the temperature values in the algorithm accordingly.

The value of the prediction horizon should also be chosen considering the dynamic response of the system to a step response: it should be long enough to cover the majority of the change in this response.

\subsection{Effect of energy price}

The simulation experiments performed in subsections 4.2 and 4.3 show that both versions of the proposed model predictive schedulers are able to decrease the energy costs in a day-ahead market environment. The simulation experiments also show that the proposed algorithms are really useful in the energy price growth periods. Of course, in price falling periods the optimization problem to be solved is trivial and in such cases there is not a big difference between the proposed ones and classical methods. The proposed MPC based scheduler is sensitive to the rapid changes in the heat capacity, in such cases (primarily

\subsection{Generalization}

The specialties discussed in the previous subsection 5.1 can be used to find other possibilities for applying the proposed adaptive cost-optimal model predictive scheduling method. These cases include the following items. 
- Multiple independent equipments

When more than one freezer operate in a household, shop or plant that are independent of each other but have the same dynamically changing electricity price, then one can optimize their operation in parallel and independently of each other to get an overall optimum in cost.

- Freezer chambers

Freezers in supermarkets or large industrial freezers are typically of distributed parameter nature, or consist of freezer chambers. These chambers have their individual cooling motor that cools their back walls, but they are connected to their neighboring chambers through their side walls. Therefore, these chambers are not independent of each other but can be regarded as a multiple-input multiple-output system with the cooling switches as inputs and chamber temperatures as outputs. Here the monotonicity conditions (ii) of subsection 5.1 should be checked that do not necessarily hold in this case.

\section{- Heaters}

Simple heaters or boilers equipped with a constant power heating device controlled by a binary switch obey both conditions of subsection 5.1 so the proposed heuristic method can be applied. However, the monotonicity conditions hold in another form: the response of the model output temperature variables is strictly monotonous for a (step) change in input such that when the switch is on, then the temperatures are increasing, otherwise decreasing.

5.4. Advantages and disadvantages of the proposed cost-optimal adaptive scheduling method

\section{Advantages}

- simple and effective, easy to use and implement even in embedded hardware thanks to the preparatory off line steps (see Section 3.4) 
- it scales well for multiple freezers/heaters that operate independently and in parallel (see Section 5.3)

- most often only a short prediction horizon (1-2 hours) are only needed, so the algorithm is essentially a real-time one, it can handle frequently changing energy price level. This property ensures that a proper control action is generated even when the energy price is not given accurately for the next 24 hours but only for the prediction horizon (1-2 hours) in a sliding window.

\section{Disadvantages}

- It uses a very simple 2 nd order model that is never accurate in practice. This is circumvented by calibrating the model, i.e. fitting the model parameters to the real dynamic response (see Section 4.1)

- the parameters of the algorithm (most importantly the sampling rate and the prediction horizon) should be determined empirically using simulation before real-time application.

\section{Conclusions and future work}

A cost-optimal model predictive scheduling algorithm was proposed in this paper that operates in a day-ahead market environment and thus it is a possible tool for demand side management. The optimization algorithm behind the method is a heuristic branch and bound algorithm that finds the cost-optimal scheduling sequence of a freezer in presence of constraints and hourly changing energy price. Since the interior air heat capacity of a freezer (or refrigerator) is

a parameter that changes during use, the proposed model predictive scheduling algorithm was extended with a conditional identification step that provides an estimate of the actual heat capacity of the controlled system.

Simulation experiments were performed on a freezer model with parameters identified from measurement data. The experiments show that the proposed cost-optimal scheduling algorithm was able to decrease the cost of operation. 
Moreover, it was shown that the prediction horizon does not affect the reached optimal cost. It is also apparent from the experiments that the computational complexity increases when the price is growing which is consistent with the engineering expectations. However, the number of price growths is more important than the value of the price. The dependence of the complexity on the parameters of the algorithms is important for a possible future micro-controller based implementation of the scheduler, since those computational platforms are able to tackle only a limited complexity.

The proposed method can be generalized for multiple home appliances of different kind, and for heating appliances, too.

\section{Acknowledgement}

This research is supported by the National Research, Development and Innovation Office - NKFIH through grant No. 115694. A. Magyar was supported by the János Bolyai Research Scholarship of the Hungarian Academy of Sciences.

\section{References}

Báez-González, P., del Real, A.J., Carlini, M.A.R., Bordons, C., 2016. Dayahead economic optimization of energy use in an olive mill. Control Engineering Practice 54, 91 - 103.

Bálint, R., Hangos, K.M., Magyar, A., 2017a. Optimal scheduling of a household refrigerator using adaptive model predictive technique, IEEE, Novi Sad, Serbia.

Bálint, R., Magyar, A., 2016. Refrigerator optimal scheduling to minimize the cost of operation. Hungarian Journal of Industry and Chemistry 44, 99-104.

Bálint, R., Magyar, A., Hangos, K.M., 2017b. Cost-optimal model predictive 455 scheduling of home appliances. IFAC PapersOnline 50, 3344-3349. 
Di Giorgio, A., Liberati, F., Canale, S., 2014. Electric vehicles charging control in a smart grid: A model predictive control approach. Control Engineering Practice 22, 147-162.

Du, P., Lu, N., 2011. Appliance commitment for household load scheduling. IEEE Transactions on Smart Grid 2, 411-419.

Elliott, M.S., Rasmussen, B.P., 2013. Decentralized model predictive control of a multi-evaporator air conditioning system. Control Engineering Practice 21, $1665-1677$.

Hangos, K., Cameron, I., 2001. Process modelling and model analysis. Academic Press, London.

Herceg, M., Kvasnica, M., Jones, C., Morari, M., 2013. Multi-parametric toolbox 3.0, in: Proceedings of the European Control Conference.

Joe-Wong, C., Sen, S., Ha, S., Chiang, M., 2012. Optimized day-ahead pricing for smart grids with device-specific scheduling flexibility. IEEE Journal on Selected Areas in Communications 30, 1075-1085.

Kim, B.Y., Oh, K.K., Ahn, H.S., 2015. Coordination and control for energy distribution in distributed grid networks: Theory and application to power dispatch problem. Control Engineering Practice 43, 21-38.

Leva, A., Piroddi, L., Felice, M.D., Boer, A., Paganini, R., 2010. Adaptive relaybased control of household freezers with on-off actuators. Control Engineering Practice 18, $94-102$.

Ma, J., Qin, S.J., Salsbury, T., 2014. Application of economic mpc to the energy and demand minimization of a commercial building. Journal of Process Control 24, 1282-1291. trol with price prediction in real-time electricity pricing environments. IEEE Transactions on Smart Grid 1, 120-133. 
Pedersen, R., Schwensen, J., Biegel, B., Green, T., Stoustrup, J., 2017. Improving demand response potential of a supermarket refrigeration system: A food temperature estimation approach. IEEE Transactions on Control Systems Technology 25, 855-863.

Pedrasa, M.A.A., Spooner, T.D., MacGill, I.F., 2010. Coordinated scheduling of residential distributed energy resources to optimize smart home energy a services. IEEE Transactions on Smart Grid 1, 134-143. doi 10.1109/TSG. 2010.2053053 .

Rodrigues, E., Godina, R., Pouresmaeil, E., Ferreira, J., Catalão, J., 2017. Domestic appliances energy optimization with model predictive control. Energy Conversion and Management 142, 402-413.

Schné, T., Jaskó, S., Simon, G., 2014. Dynamic modeling and identification of a domestic refrigerator. FUTURE INTERNET TECHNOLOGIES , 45.

Setlhaolo, D., Xia, X., 2014. Optimal scheduling of household appliances incorporating appliance coordination. Energy Procedia 61, 198-202.

Shimomachi, K., Hara, R., Kita, H., Noritake, M., Hoshi, H., Hirose, K., 2014. Development of energy management system for dc microgrid for office building:-day ahead operation scheduling considering weather scenarios, in: Power Systems Computation Conference (PSCC), 2014, IEEE. pp. 1-6.

Sossan, F., Lakshmanan, V., Costanzo, G.T., Marinelli, M., Douglass, P.J., Bindner, H., 2016. Grey-box modelling of a household refrigeration unit using time series data in application to demand side management. Sustainable Energy, Grids and Networks 5, 1-12.

Sou, K.C., Weimer, J., Sandberg, H., Johansson, K.H., 2011. Scheduling smart home appliances using mixed integer linear programming, in: 2011 50th IEEE Conference on Decision and Control and European Control Conference, pp. 5144-5149. doi 10.1109/CDC.2011.6161081. 
Spot, E., 2010. Market data. System price.[Verkkotietokanta][Viitattu 23.11. 2011] Saatavissa: http://www. nordpoolspot. com/Marketdata1/Elspot/Area-Prices/ALL1/Hourly/http://www. nordpoolspot. com/Market-data1/Elspot/Area-Prices/ALL1/Hourly .

Tianhu, M., Jumpei, B., Yumiko, I., 2017. Analysis of microgrid contributing to hour-ahead market operation through marginal day-ahead market price-based demand response. Energy Procedia 118, 119-127. 